\title{
Suppressive effects of whey protein hydrolysate on sucrose- induced hyperglycemia in silkworms
}

\author{
Yasuhiko Matsumoto, ${ }^{1,2}$, Miki Takahashi ${ }^{2,3}$, Masahiro Umehara ${ }^{4}$, Masato Asano ${ }^{4}$, \\ Hiroko Maruki-Uchida ${ }^{4}$, Minoru Morita ${ }^{4}$, Kazuhisa Sekimizu ${ }^{2,3, *}$ \\ ${ }^{1}$ Department of Microbiology, Meiji Pharmaceutical University, Tokyo, Japan; \\ ${ }^{2}$ Teikyo University Institute of Medical Mycology, Tokyo, Japan; \\ ${ }^{3}$ Genome Pharmaceuticals Institute Co., Ltd., Tokyo, Japan; \\ ${ }^{4}$ Health Science Research Center, Research and Development Institute, Morinaga and Company Limited, Kanagawa, Japan.
}

\begin{abstract}
Summary Silkworms are useful for evaluating substances that suppress postprandial hyperglycemia by oral administration. In this study, orally administered whey protein hydrolysate (WPH), obtained by enzymatic treatment of whey protein, suppressed sucrose-induced hyperglycemia in silkworms in a dose-dependent manner. WPH also inhibited glucoseinduced hyperglycemia in silkworms. These findings suggest that WPH contains a bioactive peptide that inhibits glucose uptake from the intestinal tract and thereby suppresses sucrose-induced hyperglycemia.
\end{abstract}

Keywords: Hyperglycemia, silkworm, sucrose, whey protein hydrolysate

\section{Introduction}

Sucrose is one of the main sweeteners added to various foods (1). Increased blood glucose levels due to excessive intake of sucrose induces the development of lifestyle-related diseases $(2,3)$. Therefore, the development of strategies to suppress postprandial hyperglycemia induced by sucrose intake will contribute to the maintenance of human health (4).

Blood glucose levels are regulated at various stages by the functions of several organs: enzymatic decomposition of sucrose in the intestine, absorption from the intestine, distribution and metabolism in various organs, and excretion outside the body (5). Therefore, experiments to accurately evaluate active substances that suppress sucrose-induced hyperglycemia must be performed using whole animals.

We previously reported diabetic silkworm models for evaluating anti-diabetic agents such as human insulin, pioglitazone, and metformin (6-9). Moreover, we established a silkworm model to search for substances

Released online in J-STAGE as advance publication October $14,2019$.

*Address correspondence to:

Dr. Kazuhisa Sekimizu, Teikyo University Institute of Medical Mycology, 359 Otsuka, Hachioji, Tokyo, 192-0395, Japan.

E-mail: sekimizu@main.teikyo-u.ac.jp that suppress sucrose-induced hyperglycemia (10-13). Enterococcus faecalis YM0831, a lactic acid bacteria strain that was identified using an in vivo silkworm evaluation system, suppresses sucrose-induced hyperglycemia in humans (14). Therefore, the in vivo silkworm evaluation system is useful for identifying candidate anti-diabetic drugs and foods that control blood glucose levels in humans.

Whey protein is a fermentation liquid obtained as a byproduct of yogurt or cheese production. In young adult females, whey protein suppresses sweetened beverageinduced hyperglycemia (15). In addition, whey protein hydrolysate (WPH), obtained by enzymatic treatment of whey protein, exhibits anti-oxidative properties and inhibits angiotensin-I-converting enzyme $(16,17)$. The mechanism of action of whey protein against postprandial hyperglycemia, however, is unclear.

In this study, we demonstrated that WPH suppressed sucrose-induced hyperglycemia in silkworms. To our knowledge, this is the first study demonstrating that WPH suppresses postprandial hyperglycemia in an animal model.

\section{Materials and Methods}

\subsection{Reagents}

Whey protein and WPH, prepared by enzymatic 
hydrolysis of whey protein, were purchased from Fonterra Japan (Tokyo, Japan). The protein content of the whey protein was $80 \%$ and that of the WPH was $83 \%$. High-performance liquid chromatography (HPLC) grade acetonitrile $(>99.8 \%$ ) and special grade trifluoroacetic acid ( $>98.0 \%$ ) were purchased from FUJIFILM Wako Pure Chemical Corporation (Osaka, Japan).

\subsection{Size exclusion chromatography}

Whey protein $(10.0 \mathrm{mg})$ and WPH $(9.6 \mathrm{mg})$ were suspended in ultrapure water $(2.0 \mathrm{~mL})$. The mixture was centrifuged $\left(20,400 \mathrm{~g}, 20^{\circ} \mathrm{C}, 10 \mathrm{~min}\right)$, and the supernatant was subjected to size exclusion

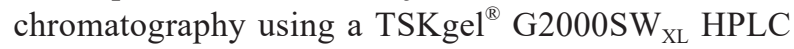
column $(300 \times 7.8 \mathrm{~mm}$ i.d., particle size: $5 \mu \mathrm{m}$, Tosoh Corporation, Tokyo, Japan) with a TSKgel ${ }^{\circledR} \mathrm{SW}_{\mathrm{XL}}$ guard column $(40 \times 6.0 \mathrm{~mm}$ i.d., particle size: $7 \mu \mathrm{m}$, Tosoh Corporation). The analysis was performed with an HPLC system (autosampler: AS-2057, pump: PU-2080, column oven: CO-2065, PDA detector: MD-2018, JASCO Corporation, Tokyo, Japan). The mobile phase was acetonitrile/water/trifluoroacetic acid $(25 / 75 / 0.1 \mathrm{v} / \mathrm{v} / \mathrm{v})$. The column oven temperature was set to $30^{\circ} \mathrm{C}$ and the flow rate was $0.6 \mathrm{~mL} / \mathrm{min}$. Absorbance at $280 \mathrm{~nm}$ was monitored. The data was analyzed using ChromNAV (version 1.18.04, JASCO Corporation).

\subsection{Silkworm rearing conditions and sucrose tolerance test}

Silkworms were reared as reported previously $(6,18)$. Sucrose or glucose tolerance tests using the silkworms were performed according to a previous report (10). Briefly, test samples were mixed with artificial diet containing $10 \%$ glucose or $10 \%$ sucrose. The diet was fed to the silkworms for $1 \mathrm{~h}$ and the glucose level in the silkworm hemolymph was measured using a glucometer (Accu-Chek, Roche, Basel, Switzerland).

\subsection{Statistical analysis}

All experiments were performed at least twice. The significance of differences was calculated using a twotailed Student's t-test. A p value of less than 0.05 was considered significant.

\section{Results}

\subsection{Characterization of whey protein and WPH by size exclusion chromatography}

We compared the size exclusion chromatography patterns between whey protein and WPH. Each sample was subjected to size exclusion chromatography. The whey protein produced major peaks at elution times ranging from 11.5 to $13.5 \mathrm{~min}$ (Figure 1). The WPH, in contrast, did not have these two major peaks, and instead multiple peaks were observed at elution times ranging from 14 to $20 \mathrm{~min}$ (Figure 1). This finding suggests that the WPH comprised whey proteins digested into low molecular mass peptides.

\subsection{Suppressive effect of WPH on sucrose-induced hyperglycemia in silkworms}

When an artificial diet containing $10 \%$ sucrose is fed to silkworms for $1 \mathrm{~h}$, the glucose level in the silkworm hemolymph increases to $300-400 \mathrm{mg} / \mathrm{dL}$ (10). In the present study, we investigated the effects of whey protein and WPH on the sucrose-induced hyperglycemia in silkworms. The blood glucose level was lower in silkworms fed the $10 \%$ sucrose diet supplemented with $10 \% \mathrm{WPH}$ than in control silkworms (Figure 2). Supplementation with acarbose, an $\alpha$-glycosidase inhibitor used as a positive control, also exhibited a suppressive effect (Figure 2). In contrast, when whey protein was added to the $10 \%$ sucrose diet, the blood glucose level of the silkworms did not decrease (Figure 2). We next examined the dose dependence of the inhibitory effect of WPH on sucrose-induced hyperglycemia in silkworms in a sucrose tolerance test. WPH in the range of $0-10 \%$ of the diet suppressed the sucrose-induced hyperglycemia in silkworms in a dose-dependent manner (Figure 3).

\subsection{Suppressive effect of WPH on glucose-induced hyperglycemia in silkworms}

To clarify the mechanism of the suppression of the

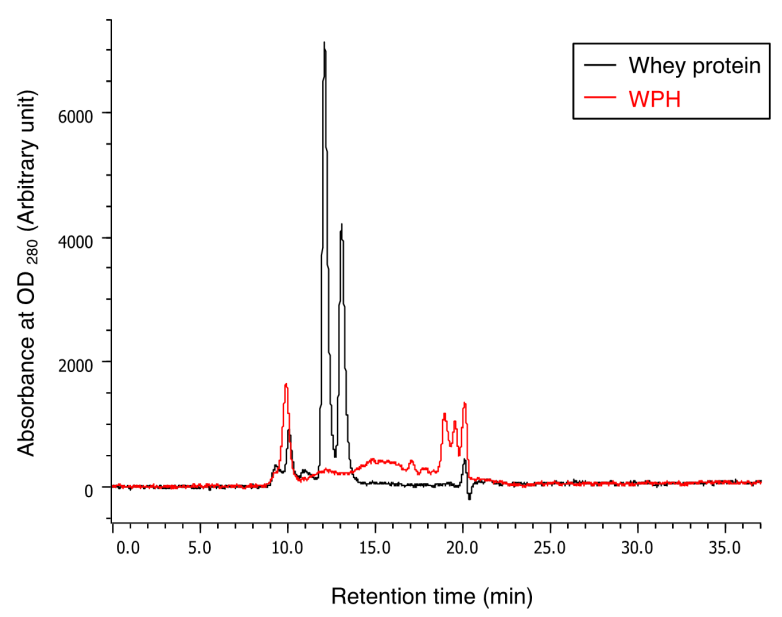

Figure 1. Molecular weight distribution by size exclusion chromatography of whey protein and whey protein hydrolysate. Whey protein $(10.0 \mathrm{mg})$ and whey protein hydrolysate (WPH, $9.6 \mathrm{mg}$ ) were suspended in $2 \mathrm{~mL}$ ultrapure water. The suspensions were centrifuged, and the supernatant was subjected to size exclusion chromatography. Absorbance at $280 \mathrm{~nm}$ was monitored. 


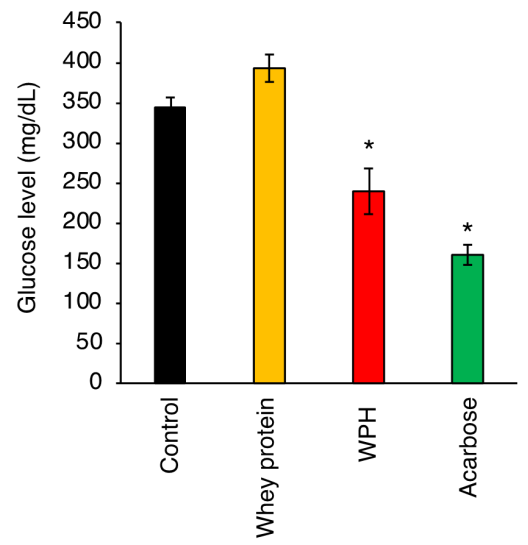

Figure 2. Effects of whey protein and whey protein hydrolysate on sucrose-induced hyperglycemia in silkworms. A $10 \%$ sucrose diet without sample (Control) or with $10 \%$ whey protein (Whey protein), whey protein hydrolysate (WPH), or 4\% acarbose (Acarbose) was fed to silkworms for $1 \mathrm{~h}$. After feeding, the blood sugar level of the silkworms was measured. Data represent mean \pm SEM. Statistically significant differences between control and other groups were evaluated using Student's $t$-test. ${ }^{*} p<0.05 . n=7$ / group.

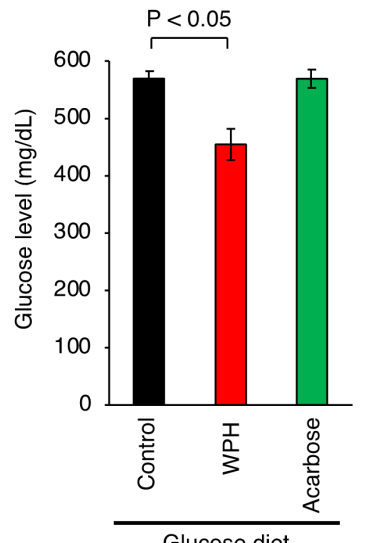

Figure 4. Suppressive effects of whey protein hydrolysate on glucose-induced hyperglycemia in silkworms. A $10 \%$ glucose diet without sample (Control) and with $7.5 \%$ whey protein hydrolysate (WPH) or 4\% acarbose (Acarbose) was fed to silkworms for $1 \mathrm{~h}$. After feeding, the blood sugar level of the silkworms was measured. Data represent mean \pm SEM. Statistically significant differences between the control and other groups were evaluated using the Student's $t$-test. $n=7 /$ group.

sucrose-induced hyperglycemia by WPH, we tested whether WPH also suppresses glucose-induced hyperglycemia. When artificial diet containing $10 \%$ glucose is fed to silkworms for $1 \mathrm{~h}$, the glucose level in the silkworm hemolymph increases to $500 \mathrm{mg} / \mathrm{dL}$ or more (10). The increase in the blood glucose level of the silkworms is not suppressed by acarbose, an $\alpha$-glycosidase inhibitor (10). The blood sugar level was lower in silkworms fed a $10 \%$ glucose diet containing $7.5 \%$ WPH than in the control silkworms (Figure 4). These findings indicate that WPH suppresses glucoseinduced hyperglycemia.

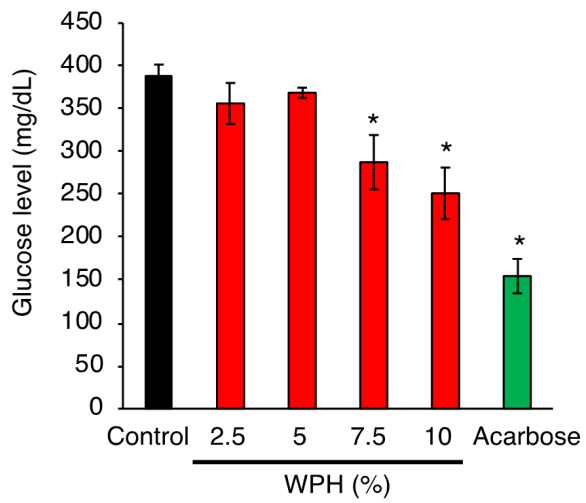

Figure 3. Dose dependence of the suppressive effect of whey protein hydrolysate on sucrose-induced hyperglycemia in silkworms. A $10 \%$ sucrose diet without sample (Control) and with $2.5,5,7.5,10 \%$ whey protein hydrolysate (WPH) or 4\% acarbose (Acarbose) was fed to silkworms for $1 \mathrm{~h}$. After feeding, the blood sugar level of the silkworms was measured. Data represent mean \pm SEM. Statistically significant differences between control and other groups were evaluated using the Student's $t$-test. ${ }^{*} p<0.05 . n$ $=4-10 /$ group

\section{Discussion}

The findings of the present study demonstrated that WPH inhibited sucrose-induced hyperglycemia in an in vivo evaluation system using silkworms, whereas whey protein did not have the same effect. Further, WPH suppressed glucose-induced hyperglycemia. This finding indicates that the anti-hyperglycemic effect of WPH cannot be explained by the inhibition of $\alpha$-glycosidase. Sucrose in the intestine is degraded to glucose and fructose by $\alpha$-glycosidase, and these monosaccharides are transferred into the blood via sugar transporters on the intestinal cells. WPH may inhibit the activity of sugar transporters responsible for the uptake of monosaccharides from the intestine into the blood. Another possibility is that WPH promotes the uptake of monosaccharides into the organs from the bloodstream.

Whey protein has an inhibitory effect on sweetened beverage-induced hyperglycemia in young adult females (15). We demonstrated that whey protein did not suppress sucrose-induced hyperglycemia in silkworms. In humans, whey protein may be degraded to WPH by proteases in the stomach and small intestine. In silkworms, whey protein may not be fully degraded to active WPH. Otherwise WPH may have a different hypoglycemic effect compared to whey protein, because it is hydrolyzed by enzyme and may have special peptides.

In conclusion, WPH has the potential to suppress postprandial hyperglycemia, but inhibition of $\alpha$-glycosidase does not explain the suppressive activity of WPH. We suggest that the use of WPH in combination with $\alpha$-glycosidase inhibitors might prevent the onset of life-related diseases such as diabetes and obesity. 


\section{Acknowledgements}

We thank Kana Hashimoto and Mari Maeda (Genome Pharmaceuticals Institute Co., Ltd, Tokyo, Japan) for technical assistance in rearing the silkworms. The project was supported by JSPS KAKENHI grant number JP15H05783 (Scientific Research (S) to KS), JSPS KAKENHI grant number JP17K08288 (Scientific Research (C) to YM), and the Supporting Industry Program by Ministry of Economy, Trade and Industry. The project was also supported by Genome Pharmaceuticals Institute Co., Ltd. (Tokyo, Japan).

\section{References}

1. Newens KJ, Walton J. A review of sugar consumption from nationally representative dietary surveys across the world. J Hum Nutr Diet. 2016; 29:225-240.

2. Johnson RJ, Nakagawa T, Sanchez-Lozada LG, Shafiu M, Sundaram S, Le M, Ishimoto T, Sautin YY, Lanaspa MA. Sugar, uric acid, and the etiology of diabetes and obesity. Diabetes. 2013; 62:3307-3315.

3. Pereira MA. Sugar-sweetened and artificially-sweetened beverages in relation to obesity risk. Adv Nutr. 2014; 5:797808.

4. Ochoa M, Lallès JP, Malbert CH, Val-Laillet D. Dietary sugars: their detection by the gut-brain axis and their peripheral and central effects in health and diseases. Eur J Nutr. 2015; 54:1-24.

5. Lewis AS, McCourt HJ, Ennis CN, Bell PM, Courtney $\mathrm{CH}, \mathrm{McKinley} \mathrm{MC}$, Young IS, Hunter SJ. Comparison of $5 \%$ versus $15 \%$ sucrose intakes as part of a eucaloric diet in overweight and obese subjects: effects on insulin sensitivity, glucose metabolism, vascular compliance, body composition and lipid profile. A randomised controlled trial. Metab Clin Exp. 2013; 62:694-702.

6. Matsumoto Y, Sumiya E, Sugita T, Sekimizu K. An invertebrate hyperglycemic model for the identification of anti-diabetic drugs. PLoS ONE. 2011; 6:e18292.

7. Matsumoto $\mathrm{Y}$, Ishii M, Ishii K, Miyaguchi W, Horie R, Inagaki Y, Hamamoto H, Tatematsu KI, Uchino K, Tamura T, Sezutsu H, Sekimizu K. Transgenic silkworms expressing human insulin receptors for evaluation of therapeutically active insulin receptor agonists. Biochem Biophys Res Commun. 2014; 455:159-164.
8. Matsumoto Y, Ishii M, Hayashi Y, Miyazaki S, Sugita T, Sumiya E, Sekimizu K. Diabetic silkworms for evaluation of therapeutically effective drugs against type II diabetes. Sci Rep. 2015; 5:10722.

9. Matsumoto Y, Sekimizu K. Evaluation of anti-diabetic drugs by using silkworm, Bombyx mori. Drug Discov Ther. 2016; 10:19-23.

10. Matsumoto $\mathrm{Y}$, Ishii M, Sekimizu K. An in vivo invertebrate evaluation system for identifying substances that suppress sucrose-induced postprandial hyperglycemia. Sci Rep. 2016; 6:26354.

11. Ishii M, Matsumoto Y, Sekimizu K. Inhibitory effects of alpha-cyclodextrin and its derivative against sucroseinduced hyperglycemia in an in vivo evaluation system. Drug Discov Ther. 2018; 12:122-125.

12. Ishii M, Matsumoto Y, Sekimizu K. Bacterial polysaccharides inhibit sucrose-induced hyperglycemia in silkworms. Drug Discov Ther. 2018; 12:185-188.

13. Ishii M, Matsumoto Y, Katada T, Sekimizu K. Additive effects of Kothala himbutu (Salacia reticulata) extract and a lactic acid bacterium (Enterococcus faecalis YM0831) for suppression of sucrose-induced hyperglycemia in an in vivo silkworm evaluation system. Drug Discov Ther. 2019; 13:133-136.

14. Matsumoto Y, Ishii M, Hasegawa S, Sekimizu K. Enterococcus faecalis YM0831 suppresses sucrose-induced hyperglycemia in a silkworm model and in humans. Commun Biol. 2019; 2:157.

15. Zafar TA, Waslien C, AlRaefaei A, Alrashidi N, Al Mahmoud E. Whey protein sweetened beverages reduce glycemic and appetite responses and food intake in young females. Nutr Res. 2013; 33:303-310.

16. Jiang B, Na J, Wang L, Li D, Liu C, Feng Z. Separation and enrichment of antioxidant peptides from whey protein isolate hydrolysate by aqueous two-phase extraction and aqueous two-phase flotation. Foods. 2019; 8:34.

17. Guo Y, Jiang X, Xiong B, Zhang T, Zeng X, Wu Z, Sun $\mathrm{Y}$, Pan D. Production and transepithelial transportation of angiotensin-I-converting enzyme (ACE)-inhibitory peptides from whey protein hydrolyzed by immobilized Lactobacillus helveticus proteinase. J Dairy Sci. 2019; 102:961-975.

18. Matsumoto Y, Sekimizu K. Silkworm as an experimental animal to research for fungal infections. Microbiol Immunol. 2019; 63:41-50.

(Received September 21, 2019; Accepted September 29, 2019) 